To be published as:

Pereira, C., Heitor, T., Heylighen, A. (2018). Improving pool design: Interviewing physically impaired architects. In: Langdon P., Lazar J., Heylighen A., Dong H. (Eds.), Breaking Down Barriers - Usability, Accessibility and Inclusive Design Springer. 


\title{
Improving pool design: Interviewing physically impaired architects
}

\author{
C.M. Pereira, T.V. Heitor and A. Heylighen
}

\begin{abstract}
People with a temporary or permanent physical impairment are often excluded from bathing activities, due to the difficulties of getting into and out of the water. This paper explores pool design, specifically the design of the access to the tank, which is key for a pool's inclusivity. In trying to break down existing barriers between users, accessibility experts and designers, we interviewed physically impaired architects about their perception of four types of pool access often used by wheelchair users; ramps, transfer walls, transfer systems and lifts. The interviews revealed limitations in all four types of pool access. To compensate for the limitations identified, combining different types of access in one single pool may be of interest. Moreover, the interviews allowed to identify another type of pool access, designed by one of the interviewees: an upper pool border connected to an underwater bed and seat allows for an easier exit than transfer walls and transfer systems. Another interviewee advanced the idea of a cane holder for physically and visually impaired people, which may contribute to freeing poolside floors from obstacles and reducing the risk of falls.

These insights may contribute to making pools more inclusive, by accommodating specific temporary or permanent mobility needs of all of us.
\end{abstract}

\section{Introduction}

Aquatic activities are considered a way of promoting health and well-being (Middlestadt et al. 2015, Sato et al. 2007). Pools have the potential of providing freedom to people with impairments, e.g. a wheelchair user or a blind person often do not need any assistive device in order to swim. Movements in the water provide freedom and facilitate physical activity, being highly beneficial for some physically impaired people. Paradoxically, non-ambulant people often cannot

Carlos Mourão Pereira, University of Lisbon, carlosmouraopereira@gmail.com; Teresa Valsassina Heitor, University of Lisbon, teresa.heitor@ist.utl.pt; Ann Heylighen, KU Leuven, ann.heylighen@kuleuven.be 
access pools autonomously due to their design. This study therefore aims to explore inclusive and multi-sensory design solutions for pool access.

For a pool access to be usable by a non-ambulant person, its design needs to facilitate mobility, centralising the effort in the upper body. As a result, it has the potential to increase haptic comfort for other users, allowing for less effort in some body parts.

Fletcher (2009) points to the fact that architects have the power to increase or decrease people's disability through their design. Unfortunately, contemporary pools where entry in and exit from the water are facilitated are rare. We argue that integrating requirements related to usability by physically impaired people may contribute to increasing haptic qualities also for users without physical impairment; e.g. a pool ramp requires less effort than a pool ladder, increasing the sensory comfort in entering and exiting the water.

This study is exploratory and its goal is to understand pool access from the perspective of diverse users, in particular those with a physical impairment. Moreover, as pools are important spaces for promoting health and well-being, making them more inclusive may increase the quality of life and reduce the need for medical care, thus contributing to a socially sustainable economy. It is important to consider that an inclusive pool is less likely to require future functional adaptations or to be demolished due to obsolescence. This makes it much more durable than a pool without inclusive access and avoids the need for further consumption of building materials, contributing to a more sustainable environment.

Current architectural production is predominantly ocularcentric, paying little attention to the spatial poetics related to integrating other sensory modalities than vision. Moreover, barrier-free spatial components are often designed with neither spatial poetics nor sensory balance in mind. As a result, functionality is perceived by most designers as something with a medical appearance, spoiling the visual poetics of the architecture. According to Guimarães (2011), inclusive sustainability requires a cultural revolution that integrates poetics into the design. Following this premise, we centred our research on the perceptions of physically impaired architects, a group with the potential to break down barriers between the poetics of designers, the inclusivity of accessibility experts and the kinaesthetic experience of physically impaired people.

Pool ramps are usually perceived as an inclusive type of access and mechanical devices as an alternative assistive solution. In order to question this perception, we set out to inventory the advantages and limitations of different types of access, which results in the identification of inclusive pool details unknown in literature.

\section{Context}

The potential to acquire spatial knowledge through the experience of disabled designers is increasingly recognised (Vermeersch \& Heylighen 2011, 2013, Pérez Liebergesell et al. 2017). According to Ostroff et al. (2002), the condition of living with an impairment can increase spatial maturity, empathy, determination and 
social justice. Regarding pool design, Usandizaga (2013) stresses that integrating inclusive premises in the early stages of the design process results in high-quality architecture, as exemplified by the works developed by disabled designers. One of these works is an unusual pool designed by one of the interviewees, which is described in more detail below.

Accessibility standards, including legislation and non-mandatory best practice guidelines, are important documents for achieving inclusivity. Usually, they are based on scientific literature and result from the confrontation with concrete realities that are inherent to the approach adopted in that context. Across the board pool standards in general, and standards on tank access in particular, are poorly developed.

The inclusive principles provided by Story et al. (1998) present a pool ramp as an inclusive spatial component related with equitable use, especially by physically impaired people or children when they are learning to swim.

The accessibility guidelines of SE (2002) recommend a pool ramp, mainly in the shape of a beach, as the best type of pool access. They mention that, for some users, a handrail along the ramp suffices, while others require assistance. These requirements reflect an awareness of the loss of autonomy of some users.

The accessibility guidelines of USAB (2004) present a potentially usable tank access, consisting of a pool lift, with the requirement that it must allow users autonomy by being operable both from the deck and from inside the pool, avoiding that users are alone in the water, waiting for assistance. These guidelines establish a minimum of one access type, specifically a pool lift or ramp, for tanks with walls with a perimeter of less than $91.44 \mathrm{~m}$. Bigger pools require an extra access type, be it a pool lift, pool ramp, pool stairs, transfer wall, or transfer system. Using different types of access is recommended in order to permit usability options for impaired users with a diversity of needs.

Transfer walls, also known as low walls, are often found in health and wellbeing centres; e.g. hot tubs. They enable people to transfer from a wheelchair to the top of the wall and rotate to the pool tank. They can be the pool edge in the case of pools with a water level over the deck, or partial pool borders accessible by a dry ramp leading to a partial low deck. In these guidelines, transfer walls require a minimum of one grab bar perpendicular to the pool wall and installed on top of the transfer wall.

The transfer system mentioned in these guidelines, also known as transfer steps or transfer tiers, combines pool stairs with a transfer platform with extra steps over the deck. This enables physically impaired people to transfer from a wheelchair to the top of the platform, and to move through the steps in a seated position when entering or exiting the tank

Howard et al. (2008) present a check-list that facilitates interpretation of the USAB (2004) guidelines. Moreover, they identify the pool lift and transfer system as the means of access that is easiest to install in existing pools.

The American legislation DJ (2010) is based on the already mentioned USAB guidelines (2004). This legislation does not guarantee pool inclusivity, mainly because it allows for pools that have only one type of access for non-ambulant people, with a pool ramp being acceptable. This ramp access affords less autonomy 
than a pool lift, and excludes people with more severe physical impairments. Still, this legislation was one of the most detailed and inclusive we found.

With regard to the requirements of DJ (2010), Caden (2011) mentions that they allow for the use of portable pool lifts. In our opinion, this condition risks the creation of more operation discontinuities. Pool lifts are less expensive to install than pool ramps, but the latter are free of the extra maintenance that a mechanical device requires (Caden 2011).

\section{Methodology}

The exploratory research approach we adopted is based on qualitative inquiry. According to Denzin (2011), current qualitative research explores the hopes and needs of a democratic society. We argue that by exploring inclusivity, the goal of this study can be representative of a democratic approach, focusing on the premise of equitable use by all people.

We interviewed physically impaired architects, combining their user experience, professional knowledge and expertise on inclusivity. Ostroff (1997) defines a user/expert as anyone who has developed natural experience in dealing with the challenges of our built environment. Confronting the user/experts' perspective is expected to contribute to spatial inclusivity. Therefore, we explore the perspective of a selective group of users with the potential of identifying inclusive design requirements for pool access.

In order to maximise cultural diversity, we recruited participants from ten countries and four continents. We interviewed ten physically impaired architects, namely Christiaan Zandstra (Netherlands), Deepak K.C (Nepal), Francesca Davenport (Australia), Gerasimos Polis (Greece), Karen Braitmayer (USA), Marcelo Guimarães (Brazil), Marta Bordas-Eddy (Spain), Nikola Arsic (Croatia), Silke Schwarz (Germany) and Yoshihiko Kawauchi (Japan).

For reasons of feasibility, we interviewed them via e-mail, an alternative qualitative interview technique applied in similar geographical contexts (Flick 2009). Moreover, given the interviewees' expertise, it is interesting to obtain written reflections, and e-mail interviews have the potential to yield more carefully considered answers. We used a semi-structured format in the questionnaire, in order to obtain selective qualitative data, without losing the opportunity to extend the interviews, some of which were developed over the course of several e-mails. The starting point was the identification of the advantages and limitations of each type of access often used by wheelchair users when entering and exiting pool tanks. We considered both the self-use by the interviewee and the allocentric perception related to inclusive use. A link to the American legislation (USAB 2004, DJ 2010) was sent to most interviewees, to help elicit responses for the interview. This legislation has been identified in our literature review as a state-ofthe-art reference, important for achieving inclusive perceptions that are pertinent for the improvement of pool design. We did not, however, send this link to the American interviewee, as she is familiar with her country's national standards. 
None of the participants required anonymization of the data. Data were analysed using coding to identify similarities and differences of opinion expressed by the interviewees, aiming to achieve generalizable statements related to the usability of pool access.

\section{Results}

The interviewees identified advantages and limitations regarding each type of pool access usable by physically impaired people.

Some interviewees preferred the pool ramp as means of access for their own use. Advantages they identified include continuous performance, autonomy in use and usability for specific users. "The ramp may work well for slow walkers who need a support mechanism to enter the pool," an interviewee stated. Other interviewees stressed its usability for people with a temporary physical impairment, and for people other than physically impaired people. In terms of limitations, an interviewee identified the discomfort of using a bathing wheelchair: "In order to use the entry ramp, I need to transfer from my wheelchair to the wheelchair for a pool which does not fit my body". Moreover, regarding its use by other people, he referred to the loss of autonomy in some situations requiring human assistance. Two interviewees mentioned the limitations for some physically impaired people, and one of them pointed out: "The entry ramp works for many but not for complete spinal cord injuries I would say. I understand it requires a waterproof wheelchair, and even if having one, once in the water it might be very difficult to move".

Pool access through a transfer system was considered by some interviewees to be an option for their own use. For one of them it allowed to avoid the inconvenience of depending on assistance. Moreover, "the trick is to design a good access point, and position it in the right place, so you can use it without having to watch out where your legs are going end up, of if you are going in some way injure yourself". Some pointed out, however, that it is usable only for specific users, with strength in their upper limbs. One of them explained: "The transfer steps require the ability to lift your body up to 6" vertically. Not everyone has that sort of arm strength". Other limitations that were identified relate to physically impaired people's well-being, as it is not as dignified as other types of pool access. One interviewee mentioned: "personally I think accessing a pool by a transfer system is humiliating. It is not a very sexy and elegant way to access a pool". He also pointed at the difficulty of using the transfer system in the event of an emergency, specifically when exiting the pool.

Some interviewees considered pool access by transfer wall to be a means of access that they themselves could use. One explained: "I prefer to use a quick, simple solution without any further human help". Another stressed the quality of autonomy in use for a specific impaired condition: "As a paraplegic, you can manage to do it by yourself, without anyone's help (which is always a plus)". Other interviewees highlighted the transfer wall's usability for a diversity of users, including those with temporary impairments, and appreciated its being 
continuously operational. However, limitations were identified as well: "Aging has been a very determining factor for me in abandoning this way of accessing to the water (...) one of the most negative things that a wheelchair user can make that is to lift his body on his arms and specially having all the weight been at the shoulders. Tendonitis and other injuries are guaranteed". Another interviewee agreed that this form of access is usable only by people with upper limb strength. Some interviewees questioned its usability when exiting the pool, and one of them said: "I'm wondering how and whom people do warn if they need help and want to leave the pool".

Regarding access by pool lift, some interviewees focused on the usability for their own purpose. Its greater usability specifically by physically impaired people with less mobility was also stressed. One of them mentioned: "It has the advantage of supporting the most significantly limited swimmers". A limitation was identified in the case of operational discontinuities. One remarked: "The pool lift has the disadvantage of being the least likely to be operational (any mechanized device is likely to fail)". Autonomy loss was also identified as a limitation: "[the pool lift] is rarely installed in a manner to be used independently". Indeed, "The main disadvantage of it is that we always need someone who is specialized for its operation. Drawing the attention of people while using it is something which I do not like at all". Another limitation is the "very slow process to use (to transfer to/from wheelchair, to operate)". Moreover, pool lifts run the risk of causing accidents: "I know hoists with chairs (...) for immersion. These chairs can be obstacles to access by other people and can cause accidents" [translated]. One interviewee identified the potential of the platform lift, i.e. a device with a bathing wheelchair for non-ambulant people, which other people can use in a standing position. Another interviewee pointed to a specific type of pool lift, a ceilingmounted hoist (Fig.1), as being the most adequate pool access for his own use: "One can enter and exit a pool without assistance. In the case of a severely physically impaired person, we can imagine that person using a life jacket together with the hoist. When it is not in use, the hoist can be put away from the pool, so that structure does not interfere with the access of other people" [translated].

Another type of pool access was explored by Bordas-Eddy in the design of a home pool in Cabrils (Fig.2). For the purposes of this study, we name this new type of pool access a transfer bed. It combines a transfer wall with an underwater bed on one side of the pool, connected in the corner to a lower underwater seat set in another transverse pool wall. Bordas-Eddy highlighted the usability provided by the underwater bed: "there's also a bench at different heights: one at $52,5 \mathrm{~cm}$ (measured from the inferior limit of the ceramic tile down the water) to seat down, and one at $22,5 \mathrm{~cm}$ to lay down. Specially the second one is very useful to get out the water". Moreover, the corner was identified as a spatial component that facilitates entry and exit, and she reflected on her experience of using the transfer bed when pregnant: "(I was heavy and it was difficult to lift my own weight). Instead the pool lift required less effort".

The importance of a storage area for assistive objects for people with reduced mobility was also identified by some interviewees. Davenport sent drawings and pictures of several bathing facilities designed under her consultancy, allowing us to gain information about an unusual detail, a cane holder for pool access (Fig. 3). 
She specified its usability: "The idea of the cane holder came about when I saw walking sticks, crutches, white canes left on the floor near steps into the pool, creating tripping hazards as well as cluttering the pool perimeter". She also mentioned the feedback on building performance: "I originally advised the architect to incorporate the cane holder in one of our earliest projects and since then it has been incorporated in the rest of the projects. In one of the projects, a cylindrical/tubular plastic cane holder was installed instead of the rings. I think the rings are better because the tubular holder has a limited depth and will require cleaning over time".

In short, some interviewees recommend providing a choice of different pool access types given the diversity of people's needs. One interviewee stated: "there have to be more than one way of opportunities for the potential users or swimmers to take advantage and make use of it."

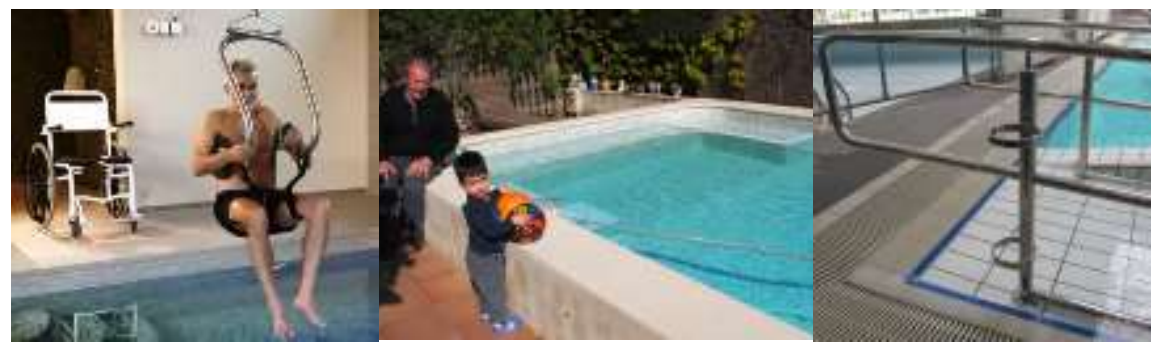

Figs. Left: 1. Ceiling-mounted pool lift; Centre: 2. Private pool in Spain with transfer bed; Right: 3. Public pool in Australia with cane holder (credits: 1. H.-M.; 2. M. B.-E.; 3. F. D.).

\section{Discussion}

Regarding the design of pool access, the standards (DJ 2010) are the most detailed legislation found in the literature. They define the criteria for accessible pools under two categories: tanks with a pool wall perimeter of over $91.44 \mathrm{~m}$ require two types of access, one of which can be a pool stair; pools with a smaller wall perimeter require only one type of access, often used by wheelchair users, specifically a ramp, a transfer system, a transfer wall or a lift. However, pool stairs can only be used by ambulant people. So, the already mentioned combination of two types of access will leave wheelchair users dependent on one of the four access types analysed. Considering that the interviewees highlighted limitations in all of the mentioned types, it is pertinent to envisage a minimum of two types of access in order to balance the limitations mentioned. Furthermore, this requirement needs to be applied to all accessible pools and not only to large tanks. Small tanks are often used for health and well-being activities, specifically in therapeutic pools for physical rehabilitation or mineral springs, and prophylactic pools at wellness centres. All of these are important facilities for the health and well-being of physically impaired people. Furthermore, equitable use and the possibility of choice for the user are principles of inclusivity (Story et al. 1998). The interviewees also stressed that mechanical devices such as a pool lift, show fewer 
limitations than non-mechanical pool accesses, in terms of their usability by people with severe physical impairment. It would be more resilient if pool standards specified the minimum provision of two types of access, one being mechanical and another non-mechanical, considering its usability by non-ambulant people.

We state that the integration of a pool lift has the potential of providing an inclusive pool, if it can be used autonomously by physically impaired people, as required by some standards (USAB 2004, DJ 2010). However, these standards recommend a pool lift with a chair, which in our opinion is a type of assistive device with avoidable medical appearance. Caden (2011) mentions that DJ (2010) standards permit the use of portable pool lifts. Several interviewees warned that these are not in continuous use. In our opinion, this situation can be improved by insisting on the installation of fixed pool lifts. Portable pool lifts are often used because they can provide access to several pools in the same facility. However, moving the lift to another pool requires assistance and means that physically impaired people have to spend time waiting. One interviewee stressed that pool lifts can cause accidents. We argue that, compared to fixed ones, movable pool lifts can increase the risk of collision with other people. It is important consider that pool decks tend to be slippery due to the presence of water. A ceiling-mounted pool lift can be an interesting user-centred option, because it reduces the risk of collision, and, aesthetically has only the presence of a ceiling rail, when not in use, which can be easily integrated into the pool design. In outdoor pools, it is more difficult to apply this solution. However, it could be interesting to design outdoor pools with partial ceiling areas to provide shade, thus more resilient to avoid ultraviolet radiation injuries.

Regarding non-mechanical pool access, the transfer bed explored by an interviewee has the potential of being more usable than the transfer wall or the transfer system. The underwater bed can be used as a rest platform, facilitating the mobility effort. Another interviewee focused on the risk of a user being in need of exiting the pool. In this situation, the rest platform can provide more safety than the transfer wall or the transfer system. Moreover, it can also be used by people without impairments, being perceived as more inclusive than the transfer wall or the transfer system. Besides enabling the seating to be used for transfer, the pool border over the deck has the advantage of providing a recognisable spatial component for visually impaired people. Furthermore, they allow for more savings in terms of volume, with fewer built areas below the deck level, and facilitate the cleaning of the pool deck, reducing the risks related with water contamination.

According to the interviewees, a pool ramp shows considerable usability limitations, because it requires a mobility effort that is impossible for some users and it has the discomfort of requiring the use of a bathing wheelchair. Also, in our perception, pool ramps may present difficulties for ambulant people, mainly cane users. This shows why a pool ramp is considered far from being an inclusive spatial component.

The findings of this research evidence that none of the analysed pool access types as such can be considered inclusive. An inclusive pool design requires different types of access, to accommodate as many needs as possible.

Storage provision, in the immediate vicinity of the pool, is required by the accessibility standards SE (2002). We argue that the requirement to provide a cane 
holder at each pool access, integrated into the proximity of handrails or grab bars, will contribute to people's safety in an inclusive way, as the ones explored by one of the interviewees.

Our findings confirm the potential of the contributions provided by disabled designers, as highlighted by several studies (Ostroff et al. 2002, Vermeersch \& Heylighen 2011, 2013, Pérez Liebergesell et al. 2017). Specifically, in the case of improving pool design, it confirms Usandizaga's (2013) observation that a physically impaired architect introduces inclusive premises in the early stage of the design process. Physically impaired architects with built works are rare. The already mentioned cases of the swimming pool with a transfer bed and the cane holders, both explored by interviewees, are exceptions and express the premise of inclusivity.

In our opinion, direct commissions and invited competitions to physically impaired architects may result in more inclusive built spaces, with the potential of inspiring other architects to achieve user-centred design solutions.

We argue that a sensory awareness of the potential of spaces usable by physically impaired people can increase comfort, allowing less effort in complex actions, such as entering and exiting the water, for people without impairment, including children and older people.

\section{Conclusion}

Our findings question the inclusivity of the most advanced legislation standards on pool design, regarding the means of entering and exiting the water. For all the types of pool access analysed, physically impaired architects identified limitations that compromise inclusivity. Therefore, we conclude that, for a pool to be inclusive, it will require a minimum of two different types of access, one being non-mechanical and another mechanical, allowing for their continuous autonomous use by non-ambulant people.

Moreover, the findings evidenced the potential of a fixed mechanical access, specifically the ceiling-mounted pool lift. Furthermore, they revealed the transfer bed as a non-mechanical access type, requiring less effort in exiting the water than the transfer wall or the transfer system, and showing two advantages compared to the ramp: it occupies less space and eliminates the discomfort of having to use bathing wheelchairs.

Furthermore, we highlight the potential of integrating cane holders at all pool access points in order to reduce the risks of falls.

This study adopted an exploratory approach to the improvement of inclusive pool design. The findings presented synthesise observations from individual interviews. In future research it may be useful to discuss them in a focus group interview. It is also important to study the types of tank access mentioned in this study by conducting walkthrough interviews with a diverse sample of users, including people with cognitive, hearing and visual impairments. 


\section{Acknowledgements}

This research was supported by Fundação para a Ciência e a Tecnologia, reference SFRH/BPD/94371/2013, with joint funding from Portugal and the European Union. We are grateful to Isabella Steffan, Ivor Ambrose, Ljerka Gordić, Sonia Carpinelli and Valerie Fletcher, for their contribution to the interviewees sample, and David M. Correia and Pedro O. Teixeira, for the visual assistance to the first author, a blind person.

\section{References}

Caden J (2011) How to Apply the American's with Disabilities Act. World Aquatic Health Conference, Colorado Springs, CO: National Swimming Pool Foundation

Denzin NK, Lincoln Y (2011) The SAGE Handbook of Qualitative Research. London: SAGE

DJ (2010) 2010 ADA Standards for Accessible Design. Washington: Department of Justice

Fletcher V (2009) Universal Design and multi-sensory environments. Art Beyond Sight Conference, New York: Metropolitan Museum of Art (oral presentation)

Flick U (2009) An Introduction to Qualitative Research. London: SAGE

Guimarães MP (2011) Writing Poetry Rather Than Structuring Grammar: Notes for the Development of Universal Design in Brazil. In: Preiser WFE, Smith KH (eds.), Universal Design Handbook. (pp. 14.1-14.9). New York: McGraw-Hill

Howard L, Young, LC, Figoni, SF (2008) Removing Barriers to Health Clubs and Fitness Facilities, Raleigh: The Center for Universal Design

Middlestadt SE, Anderson A, Ramos WD (2015) Beliefs about using an outdoor pool: Understanding perceptions of place in the context of a recreational environment to improve health. Health \& Place 34, 1-8, Amsterdam: Elsevier

Ostroff E (1997) Mining our Natural Resources: The User as Expert. Innovation, 16, 1

Ostroff E, Limont M, Hunter DG (2002). Building a World Fit for People: Designers with Disabilities at Work. Boston: Adaptive Environments Centre

Pérez Liebergesell N, Vermeersch PW, Heylighen A (2017) Disability experience through the body of a designer: the case of architect Marta Bordas Eddy. The Art of Belonging International Disability Studies Conference, Amsterdam

Sato D, Kaneda K, Wakabayashi H, Nomura T (2007) The Water Exercise Improves Health Related Quality of Life of Frail Elderly People at Day Service Facility. Quality of Life Research, 16, 10 (Dec.), 1577-1585

SE (2002) Access for Disabled People. London: Sport England

Story MF, Mueller JL, Mace R (1998) The Universal Design File: Designing for People of All Ages and Abilities. Raleigh: The Center for Universal Design

USAB (2004) Americans with Disabilities Act and Architectural Barriers Act Accessibility Guidelines. Washington: United States Access Board

Usandizaga M (2013) Learning about universal design: An experience. In: Bordas-Eddy M (ed.) Let's Open Cities for Us - LOCUS (pp. 17-23) Barcelona: Universitat Politècnica de Catalunya

Vermeersch PW, Heylighen A (2011) Scaling Haptics - Haptic Scaling. Studying scale and scaling in the haptic design process of two architects who lost their sight. In G. Adler et al. (eds.), Scale: Imagination, Perception and Practice in Architecture (pp. 127-135). Oxon: Routledge

Vermeersch PW, Heylighen A (2013) Rendering the tacit observable in the learning process of a changing body. In N. Nimkulrat et al. (eds.). Knowing Inside Out (pp. 259-270). Loughborough: Loughborough University 